\title{
ROLE OF FAVIPIRAVIR IN COVID-19: A REVIEW
}

\author{
Dhanashree Rajhans \\ Assistant Professor, Shree Gorksha College of \\ Pharmacy And Research Centre, Aurangabad, \\ Maharashtra, India.
}

\begin{abstract}
An outbreak of severe acute respiratory syndrome coronavirus 2 (SARS-CoV-2) infection have been reported in China since December 2019. Recent global outbreak of the pandemic caused by coronavirus emphasizes the urgent need for novel antiviral therapeutics. Covid-19 infection caused by the novel coronavirus SARS-COV-2 continues to be a major global health challenge. A number of medications have been proposed and there are ongoing clinical trials around the world to search a suitable treatment. Herein, we reviewed the effects of Favipiravir for the treatment of COVID-19. This review elaborates the mechanism of action, development, pharmacokinetics and details of clinical trials for Favipiravir to be beneficial and actively involved in controlling the pandemic and to provide therapeutics for the treatment of COVID-19 infection. Favipiravir shows better therapeutic responses on COVID-19 in terms of disease evolution and viral clearance. The preliminary clinical results provide useful information of treatments for SARS-CoV-2 infection. Favipiravir may be safe and tolerable in short-term use, but further evidence is needed to assess the longe-term effects of treatment.
\end{abstract}

Keywords - COVID-19, Favipiravir, clinical trials.

\section{INTRODUCTION}

In December 2019, novel coronavirus reported firstly in Wuhan, China. After the outbreak of this novel coronavirus throughout the world, WHO officially named this disease as COVID-19. (L. Wang, Y. Wang, D. Ye et.al (2020). The worldwide outbreak of the severe and acute respiratory coronavirus disease (COVID-19) caused by coronavirus strain SARS-CoV-2 is at present an important point of discussion due to the suffering this disease is causing to humankind. (Maria A. Amesty (2020))

International Committee on Taxonomy of Viruses named this newly reported disease as a severe acute respiratory syndrome coronavirus-2 (SARS-CoV-2). SARS$\mathrm{CoV}-2$ can be transmitted rapidly from person to person. (C.C. Lai, T.P. Shin, W.C. Ko et.al (2020). Some previous pathological conditions of the patients may have a major impact on survival. (Tarek Mohamed Abd et al (2020)) The

\author{
Manali Atre \\ M pharmacy Student of Y.B. Chavan College of \\ Pharmacy, Dr. Rafiq Zakaria Campus, Aurangabad, \\ Maharashtra, India.
}

coronavirus family divided into four types $\alpha-\mathrm{CoV}, \beta-\mathrm{CoV}, \gamma-$ $\mathrm{CoV}$ and $\delta-\mathrm{CoV}$. The $\alpha$ and $\beta-\mathrm{CoV}$ type mostly infects mammals where $\gamma$ and $\delta$ - CoV type infects bird \& animals. (Yang. RongGuo et.al (2020). SARS- CoV-2 is an enveloped RNA virus (N Lian et.al (2020).

As the COVID-19 is a new pandemic disease. There is no specific effective treatment for COVID-19. Many researchers were focus on many anti-viral drug candidates. Favipiravir is one of the anti-viral drug that gain attention by the researchers for the treatment of COVID-19. So here we review its action, efficacy \& safety.

\section{FAVIPIRAVIR}

Favipiravir (Avigan, T-705) is used as an anti-influenza drug. Favipiravir is a pyrazinecarboxyamide derivative having IUPAC name 6-fluro-3-hydroxypyrazine-2-carboxy-amide. Favipiravir also has an activity against viral infections like arenovirus, bunyvirus, flavivirus, alphavirus and norovirus. (i Gua et.al (2019).

\section{A. Structure of Favipiravir-}

Favipiravir function as purine analogue. Favipiravir has braod spectrum activity against RNA viruses only. It does not have any activity against DNA viruses. (Suranjith L. Senevirantne et.al (2020).

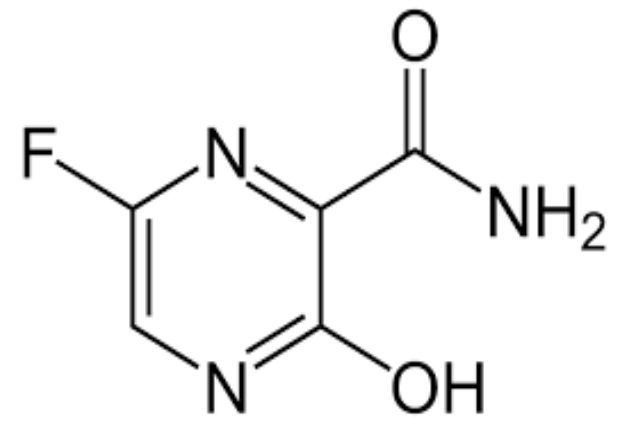

Fig. 1. Structure of Favipiravir 


\section{B. Mechanism of Action of Favipiravir:}

Favipiravir selectively inhibit RNA- dependent RNA polymerase (RdRp) of RNA virus. (Kaddoura et.al(2020). Favipiravir enters into the infected cell through the process of endocytosis and then transferred into its phosphorylated active form Favipiravir ribofuranosyl phosphate by phosphorylation. (Renyi Wu et.al (2020). During the viral RNA replication process the active form of Favipiravir inhibits the elongation of RNA strand by competing with purine nucleoside for RdRp and reduces the efficacy of replication

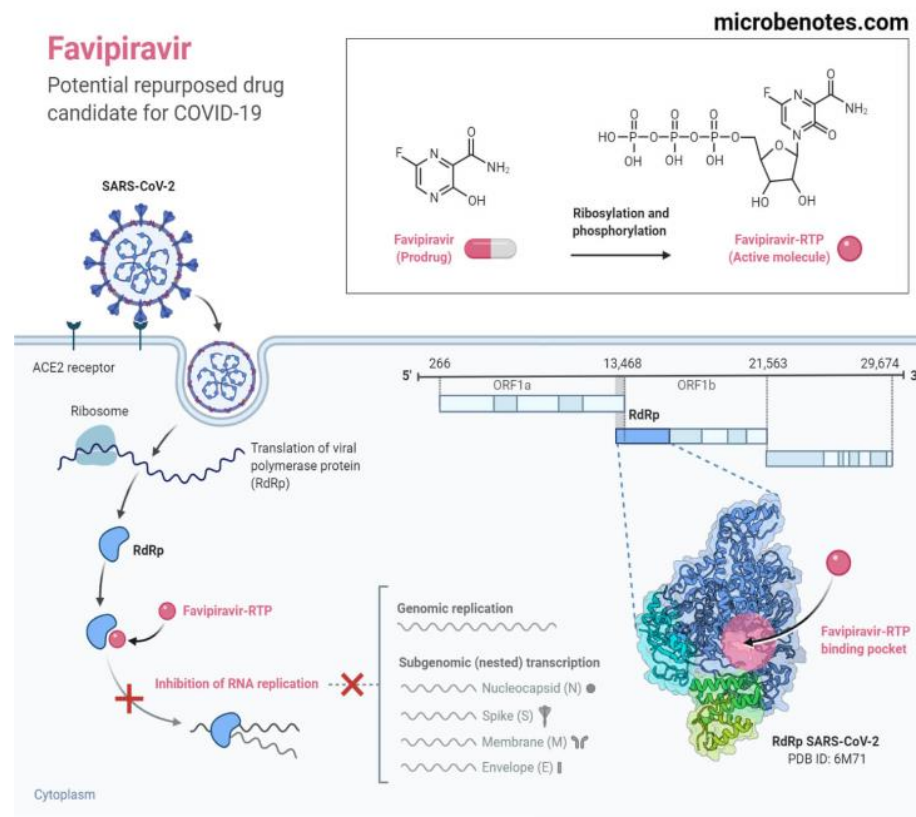

Fig. 2. Mechanism of action of Favipiravir

\section{Development of Favipiravir:}

Toyama Chemical Co., Ltd., initially developed Favipiravir (T-705) which showed anti-influenza virus activity from approximate 30,000 screened compound. After that Favipiravir was tested for its safety and efficacy. Laboratory conducted test on mice \& ferrets, compared efficacy with oseltamavir. Clinical trials were performed in Japan and U.S. (K. Shiraki, T. Daikoku et.al (2020). According to the result of efficacy, safety and action of Favipiravir in 2014, Japan Pharmaceutical and Medical device Agency was approved this drug for the treatment of influenza A virus. (Arab Zozani M. et.al (2020).

After the many studies over other RNA viruses, Favipiravir has been developed as broad spectrum anti-RNA virus drug. An anti-RNA virus activity and efficacy studies of Favipiravir was performed on animal model. In West Africa, 2014 Favipiravir was analysed for Ebola virus infections. Favipiravir has been shown to be effective against Ebola virus and influenza virus infection. (K. Shiraki, T. Daikoku et.al (2020)

After the outbreak of COVID-19, in March 2020 the National Medical Product Administration of China approved Favipiravir as the first anti-COVID- 19 drug in China because of its minimal side effect and greater efficacy. (Yanai et.al (2020)

\section{Pharmacokinetics of Favipiravir:}

The oral administration of Favipiravir on healthy Japanese volunteers showed that highest plasma concentration occurred at 2 hours and then decreased rapidly with half life time of 2 5.5 hours. Favipiravir drug undergoes metabolism in liver by the enzyme aldehyde oxidase (AO) and xanthine oxidase produces inactive metabolite (T-705M1) which is excreted by kidney. Intravenous administration of Favipiravir in cynomolgus macaques shows non-linear pharmacokinetics over time and over range of dose and continuous decline in plasma concentration after 7 days of continuous administration. From the clinical study of China showed that the dose regimen of 3,200 $\mathrm{mg}$ (1,600 $\mathrm{mg}$ twice daily) loading dose on day 1 followed by 1,200 mg maintenance dose (600 $\mathrm{mg}$ twice daily) on day 2 to day 14 is effective. (Y.X. Du, X.P Chen et.al (2020)

\section{ClinICAL STUDIES OF FAVIPIRAVIR FOR COVID-19:}

Favipirvair consider the one of the potential candidates for the SARS- CoV 2treatment. In an open label controlled trial, out of 80 patients of COVID-19, 35 patients gave oral Favipiravir plus interferon by aerosol inhalation and other 45 patients were gave lopinavir/ritonavir plus interferon by aerosol inhalation. In this study patients showed significant improvement rate of $91.43 \%$. Favipiravir showed higher improvement rate in chest imaging.(SMMSEK and UNAL et.al)

By ongoing clinical trials in China, it was reported that Favipiravir treatment showed shorter viral clearance time as compared to untreated patients. In Japan and Indonesia Favipiravir is currently used for the treatment of COVID19.(Andri Frediansyah et.al (2020)

In case report study, on young healthy male with mild COVID-19 who received supportive care and that supportive care showed tendency to improve fever reduction and negative PCR found in 20days of illness. The second case showed 60 year old man with hypertension and diabetes mellitus admitted with severe COVID-19 who received supportive care with Favipiravir. From starting the drug, temperature decreased and improvement in oxygenation and dietary intake noted. In this study, in COVID-19 patient with hypoxemia Favipiravir showed promising effect but in healthy young patient who received supportive care showed spontaneous remission in illness. (Jomah S et.al (2020)

Randomised controlled trials were conducted on 236 patients with mixed-severity of COVID-19 illness. In this study, Favipiravir was compared with umifenovir. This study 


\section{International Journal of Engineering Applied Sciences and Technology, 2020 \\ Vol. 5, Issue 4, ISSN No. 2455-2143, Pages 551-554 \\ Published Online August 2020 in IJEAST (http://www.ijeast.com)}

reported a possible increase in clinical recovery at day 7 with Favipiravir.(W. Liu, P. Zhou, K. Chen et.al (2020)

The non-randomized and randomized controlled clinical trial are conducted to investigate efficacy and safety of Favipiravir alone or in combination with tocilizumab or cholroquine phosphate. These trials are currently ongoing trials.

Glenmark Pharmaceuticals has announced top-line results from a Phase 3 clinical trial in mild to moderate COVID-19 patients conducted across seven clinical sites in India. The open-label randomised, multicentre clinical trial, conducted in 150 patients, evaluated the efficacy and safety of Favipiravir plus standard supportive care (Favipiravir treatment arm), versus standard supportive care alone (control arm), in mild to moderate patients, randomised within a 48-hour window of testing RT-PCR positive for COVID-19. Patients in the Glenmark Favipiravir clinical trial received Favipiravir tablets $3,600 \mathrm{mg}$ (Day 1) $+1,600 \mathrm{mg}$ (Day 2 or later) for up to maximum of 14 days, along with standard supportive care.

Key secondary outcome measures for clinical improvement demonstrated the efficacy and advantage of Favipiravir treatment arm over the control arm. Additionally, Glenmark's Favipiravir was well tolerated with no serious adverse events or deaths in the Favipiravir treated arm. The most commonly observed $\mathrm{AE}$ was asymptomatic transient increases in uric acid; most resolved on first follow up. Gastrointestinal disturbance was minimum and no clinically significant differences were observed between the treatment groups. (expresspharma.in (2020).

\section{CONCLUSION}

The COVID-19 pandemic shows greatest health crises in the world, all the researchers, pharmaceutical companies and biotechnology companies are involve in developing potential therapies and vaccines. Repurposing of an effective drug candidates promises the fastest therapeutic means for this pandemic. Favipiravir is one of the repurposing drug candidates having the $\operatorname{RdRp}$ antiviral drug which shows efficacy. The clinical data suggest that therapeutic action, safety and efficacy of Favipiravir in mild to moderate COVID19 patients. But ongoing clinical trials of favpiravir will provide more clarity for the use of Favipiravir against the global pandemic COVID-19.

\section{Acknowledgement:}

We acknowledge the efforts of all the researchers, medical staff and communities around the world towards reducing the impact of COVID-19 on vulnerable populations. Author's also want to thanks to the reviewers for their helpful suggestions.

Funding: No funding received.

\section{REFERENCE}

[1] Wang L., Wang Y., and Ye D.(2020). Review of the 2019 novel coronavirus(SARA-CoV-2) based on current evidence,https://doi.org/10.1016/j.ijantimicag.2020.10594 $8,($ Pg 1-7)

[2] Amesty M., Alio J.(2020). COVID-19 disease and ophthalmology: An update, https://doi.org/10.1007/s40123-020-00260-y,(Pg 1-12)

[3] Mohamed T., Stockanda J.(2020). Recent progress and challenges in drug development against COVID-19 coronavirus (SARS-CoV-2) - an update on the status, $(\mathrm{Pg}$ $1-10)$

[4] Lai C., Shih T., and Ko W.(2020). Severe acute respiratory syndrome coronavirus 2(SARA CoV-2) and coronavirus disease-209(COVID-19):The epidemic and thechallenges,https://doi.org/10.1016/j.ijantimicag.2020.1 05924,(Pg 1-9)

[5] GuoY.et al,(2020). The origin, transmission and clinical therapies on coronavirus disease 2019 (COVID-19) outbreak- an update on the status,https://doi.org/10.1186/s40779-020-00240-0,(Pg 110)

[6] Lian N., Xie H., Lin S., Huang J., Zho J., and Lin Q.(2020). Umifenovir treatment is not associated with improved outcomes in patients with coronavirus disease 2019: a retrospective study, https://doi.org/10.1016/j.cmi.2020.04.026,(Pg 917-921)

[7] Guo Q., Xu M., Guo S., Zhu F., Xie Y., and Shen J.(2019). The complete synthesis of favipiravir from 2aminopyrazine,https://doi.org/10.1007/s11696-018-06549,(Pg 1043-1051)

[8] Seneviratne S., Abeysuriya V., Mel S., Zoysa I., and NiloofaR.(2020). Favipiravir in covid-19, IJPSAT,(Pg 143-145)

[9] Wu R., Wang L., Kuo H., Shannar A., Peter R., Chou P., Shanyi L., Hudlikar R., Liu X., Liu Z., Poiani G., Amorosa L., Luigi B., and Kong A.(2020). An update on current therapeutic drug treating COVID19,https://doi.org/10.1007/s40495-020-00216-7,(Pg 5670)

[10] Kaddoura M., Albrahim M., Hijazi G., Soudani N., Auddi A., Alkalamouni H., Eid A., and Zaraket H.(2020). 
Covid-19 therapeutic options under investigation, doi:10.3389/fphar.2020.01196,(Pg 1-24)

[11] Shiraki K., and DaikokuT.(2020). Favipiravir, an antiinfluenza drug against life threatening RNA virus infections,https://doi.org/10.1016/j.pharmthera.2020.1075 $12,(\operatorname{Pg} 1-15)$

[12] Zozani M., Hassanipour S., and Nejad D.(2020). Favipiravir for treating patients with novel coronavirus (COVID-19): protocol for systematic review and metaanalysis of randomized clinical trials, doi:10.1136/bmjopen-2020-039730,(Pg 1-3)

[13] YananiH.(2020). Favipiravir: A possible pharmaceutical treatment for COVID-19, https://doi.org/10.14740/jem645,(Pg 1-3)

[14] Du Y., and Chen X.(2020). Favipiravir: Pharmacokinetics and concerns about clinical trials for 2019- $\mathrm{nCoV}$ infection, https://doi.org/10.1002/cpt.1878,(Pg 242-247)

[15] Yavuz S., and UnalS.(2020). Antiviral treatment of COVID-19, doi:10.3906/sag-2004-145,(Pg 611-619)

[16] Frediansyah A., Tiwari R., Khan S., Dhama K., and HarapanH.(2020). Antivirals for COVID-19: A critical review,https://doi.org/10.1016/j.cegh.2020.07.006,(Pg 19)

[17] Jomah S. et al,(2020). Clinical efficacy of antivirals against novel coronavirus(COVID-19): A review, https://doi.org/10.1016/j.jiph.2020.07.013,(Pg 1-9)

[18] Liu W., Zhou P., Chen K., Ye Z., Liu F., Li X., He N., Wu Z., Zhang Q., Gong X., Tang Q., Du X., Ying Y., Xu X., Zhang Y., Liu J., Li Y., Shen N., Couban R., Quazi I., Guyatt G., and Zhai S.(2020). Efficacy and safety of antiviral treatment for COVID-19 from evidance in studies of SARS-CoV-2 and other acute viral infections: a systematic review and meta analysis, doi:10.1503/cmaj.200647,(Pg E734- E744)

[19] Santos W.(2020). Natural history of COVID-19 and current knowledge on treatment therapeutic options,https://doi.org/10.1016/j.biopha.2020.110493,(Pg 1-18)

[20] https://www.expresspharma.in/covid19updates/glenmarkannounces-top-line-results-from-phase-3-clinical-trial-offavipiravir-for-covid-19-treatment. 